\title{
Geographical variation of the genes controlling hybrid breakdown and genetic differentiation of the chromosomal regions harboring these genes in Asian cultivated rice, Oryza sativa $\mathrm{L}$.
}

\author{
Shuichi Fukuoka ${ }^{1,2, *}$, Hyoji Namai ${ }^{2}$, and Kazutoshi Okuno ${ }^{1}$ \\ ${ }^{1}$ Department of Genetic Resources I, National Institute of Agrobiological Resources, Kannondai 2-1-2, \\ Tsukuba, Ibaraki 305-8602, and \\ ${ }^{2}$ Institute of Agriculture and Forestry, University of Tsukuba, Tennodai 1-1-1, \\ Tsukuba, Ibaraki 305-0006, Japan
}

(Received 27 July 1998, accepted 4 September 1998)

\begin{abstract}
The geographical variation of two genes, $h w d 1$ and $h w d 2$, controlling hybrid breakdown $\left(\mathrm{F}_{2}\right.$ weakness) was surveyed in 239 Asian rice cultivars. The cultivars with the genotype Hwd1 / Hwd1 Hwd2 / Hwd2 were predominant in South Asia and Southeast Asia, while those with the genotype $H w d 1 / H w d 1 ~ h w d 2 / h w d 2$ were predominant in Japan, the Philippines, and Yunnan province, China. Only one cultivar from Laos had the genotype $h w d 1 / h w d 1 H w d 2 / H w d 2$. Preferential association was found between the presence or absence of $h w d 2$ and the two major cultivar groups (indica and japonica) based on diagnostic RFLP markers. Dominant homozygotes were frequent in indica rice cultivars, while recessive homozygotes were frequent in japonica rice cultivars. This association was not clear for cultivars from the center of genetic diversity of rice, ranging from Vietnam to Bangladesh. Polymorphisms were surveyed for RFLP markers on chromosomes 10 and 7, harboring hwd1 and $h w d 2$ loci, respectively. The region around $h w d 2$ tended to be differentiated in allelic combination among cultivars into the indica and japonica rice groups. No such trend was found around the hwd1 region. These facts suggest the hybrid breakdown controlled by this genic system is a rare phenomenon and consequently did not contribute to cultivar differentiation of rice.
\end{abstract}

\section{INTRODUCTION}

Reproductive barriers are essential for maintaining the identity of species. The mechanisms of this phenomena in interspecific hybrids and their evolutionary roles have been discussed by many researchers and were reviewed by Stebbins (1950). Reproductive barriers observed in hybrids and their progenies were classified into three types (Stebbins, 1950). They are hybrid inviability (or weakness) and hybrid sterility observed in $\mathrm{F}_{1}$ hybrids, and hybrid breakdown observed in the $\mathrm{F}_{2}$ or later generations. Hybrid breakdown is expressed as sterility or weakness or both.

In rice the above three types of reproductive barriers have been reported by many workers (Oka, 1957a,b; Chu and Oka, 1972; Oka, 1974; Sano et al., 1979; Sato et al., 1984; Okuno, 1985; Sato and Morishima, 1988; Ise et al., 1992). Hybrid inviability (or weakness) and hybrid sterility have been widely observed both in inter- and intraspecific crosses. Hybrid breakdown has been reported only in intraspecific

\footnotetext{
* Corresponding author.
}

crosses and its evolutionary role remains unclear.

The significance of reproductive barriers in genetic differentiation depends on the frequency of its occurrence and its selective advantage. Although many genes for hybrid sterility have been reported in rice, the geographical variation of these genes have not been analyzed due to the difficulty in identifying the genes. On the other hand, the geographical variation of the genes causing $\mathrm{F}_{1}$ hybrid weakness was analyzed in inter- and intraspecific crosses of rice (Sato and Hayashi, 1983; Sato and Morishima, 1987). Those studies revealed that $\mathrm{F}_{1}$ hybrid weakness is a rare phenomenon and consequently that it can not be considered to be important in differentiation of rice. The distribution of the genes causing hybrid breakdown $\left(\mathrm{F}_{2}\right.$ weakness) have not yet been analyzed in detail.

The genetic differentiation of the chromosomal region harboring the gene responsible for reproductive barriers is an important factor to consider in relation to its distribution. Sato and Hayashi (1983) reported that the differential distribution of the alleles at the locus Hwc2 (formerly designated $L-2-a$ ), one of the complementary genes caus- 
ing $\mathrm{F}_{1}$ weakness, between indica rice $(h w c 2)$ and japonica rice $(H w c 2)$. Genes which confer selective advantage have been suggested to have an influence on the distribution of the genes linked to them. The genetic differentiation of the chromosomal region harboring the genes responsible for hybrid breakdown should be analyzed to determine the evolutionary importance of the region.

Hybrid breakdown ( $\mathrm{F}_{2}$ weakness) observed in a cross between the Japanese cultivar Sasanishiki and Thai cultivar Col. No. 15 was first reported by Okuno (1985). The weak plants are significantly shorter in plant height and have fewer tillers than normal plants. Since the weak plants cannot be distinguished from normal plants before transplanting, this phenomenon seriously reduces the number of plants available for selection in cross breeding.

The genetic basis of this hybrid breakdown, in which about $30 \%$ of the $\mathrm{F}_{2}$ population were weak, was explained by assuming a set of genes $h w d 1$ and $h w d 2$ (Okuno, 1985). We mapped the genes $h w d 1$ and $h w d 2$, using RFLP markers on chromosomes 10 and 7, respectively (Fukuoka et al., 1998). Test crosses were carried out for 100 Asian cultivars using tester lines recessive at both loci (Okuno, 1986). In that study, although a geographical difference was observed in the frequency of the genotypes, the genotypes of $70 \%$ of the cultivars were not determined. Determining the geographical distribution of these genotypes is useful for understanding the significance of these genes in the intraspecific differentiation of Asian rice cultivars and for carrying out efficient cross breeding.

In this study, 239 Asian cultivars were investigated for their gene composition, regarding $h w d 1$ and $h w d 2$ by crossing with three testers, and the geographical variation of their genotypes was examined. Further, genetic diversity in the chromosomal regions harboring $h w d 1$ and $h w d 2$ was evaluated using RFLP markers linked to these genes.

\section{MATERIALS AND METHODS}

Cultivars used and genetic characterization. A total of 239 rice cultivars from 18 Asian countries and regions (Table 1) were used to determine the genotypes for the genes controlling hybrid breakdown, $h w d 1$ and $h w d 2$. These cultivars were characterized by RFLP markers. RFLP markers are useful for the classification of rice and several individual RFLP markers can be used to classify rices into indica and japonica, ecogeographical races (Zheng et al., 1994; Qian et al., 1995; Li et al., 1998). The RFLP markers diagnostic for the cultivar groups were screened from the markers on the rice RFLP genetic linkage map (Kurata et al., 1994), using a core collection of 130 rice cultivars whose cultivar groups had been identified (data are not shown). Three RFLP markers, C601, G187, and C711, selected as diagnostic markers for distinguishing between indica and japonica rices, are located on chromosomes 2, 8, and 9, respectively. Cultivars with the alleles common to the typi-
Table 1. The number of cultivars from 18 Asian countries and areas used for this study and cultivar groups they belong to

\begin{tabular}{clrrrr}
\hline \hline & & \multicolumn{4}{c}{ Cultivar group* } \\
Code No. & Country/Area & I & II & III & total \\
\hline 1 & Sri Lanka & 11 & 1 & 1 & 13 \\
2 & India & 9 & 0 & 2 & 11 \\
3 & Bangladesh & 12 & 0 & 2 & 14 \\
4 & India (Assam) & 16 & 0 & 1 & 17 \\
5 & Nepal & 10 & 2 & 0 & 12 \\
6 & Bhutan & 4 & 9 & 0 & 13 \\
7 & Myanmar & 12 & 1 & 0 & 13 \\
8 & Thailand & 5 & 6 & 1 & 12 \\
9 & Cambodia & 11 & 0 & 0 & 11 \\
10 & Laos & 5 & 6 & 0 & 11 \\
11 & Vietnam (northwest) & 2 & 9 & 0 & 11 \\
12 & Malaysia & 6 & 6 & 0 & 12 \\
13 & Indonesia & 5 & 7 & 2 & 14 \\
14 & Philippines & 4 & 13 & 1 & 18 \\
15 & China (Yunnan) & 5 & 8 & 0 & 13 \\
16 & China (south) & 13 & 2 & 0 & 15 \\
17 & China (north) & 7 & 8 & 0 & 15 \\
18 & Japan & 0 & 14 & 0 & 14 \\
& total & 137 & 92 & 10 & 239 \\
\hline
\end{tabular}

* Cultivar group was determined based on three diagnostic RFLP markers; C601, G187, and C711. Group I and II corresponds to indica and japonica, respectively. Group III is an intermediate type based on the three RFLP markers.

cal indica type at all three loci were classified as group I, and those with the alleles common to the typical japonica type at all three loci as group II. Group III consisted of intermediate cultivars which did not fall into group I nor group II.

Test crosses. To determine the genotypes of cultivars with regard to hybrid breakdown genes, they were crossed with three testers: a double recessive tester line (either of W26, W2, or W3; hwd1/hwd1 hwd2/hwd2), Sasanishiki (Hwd1/Hwd1 hwd2/hwd2) and Col. No. 15 (hwd1/hwd1 Hwd2/Hwd2) (Okuno, 1985). The system of genotype determination is summarized (Table 2). Plants with the genotypes hwd1 / hwd1 hwd2 / Hwd2, hwd1 / Hwd1 hwd2 / $h w d 2$, and $h w d 1$ / hwd1 hwd2 / hwd2 showed weakness and plants of other genotypes showed normal plant growth (Okuno, 1985). When a cultivar to be tested has the genotype Hwd1 / Hwd1 Hwd2 / Hwd2, $\mathrm{F}_{1}$ plants from a cross with a tester line (W26, W2, or W3) are expected to have normal height and normal number of tillers, while a cultivar which is Hwd1 / Hwd1 hwd2 / hwd2 or hwd1 / hwd1 Hwd2 / Hwd2 produces weak $\mathrm{F}_{1}$ plants from crosses with the same tester line.

In the crosses with the tester cultivars, Sasanishiki and Col. No. 15, whether normal and weak plants segregate in a ratio of $11: 5$ in the $\mathrm{F}_{2}$ generation depends on the genotype of the cultivar tested. When weak plants appear in the cross with either Sasanishiki or Col. No. 15, the cultivar tested is regarded as having a recessive allele at the locus harboring dominant alleles in the tester cultivar. Cultivars whose genotypes could not be determined with this system 
Table 2. Summary of expected results of three test crosses for the three genotypes at the hwd1 and $h w d 2$ loci in Asian rice cultivars

\begin{tabular}{|c|c|c|c|}
\hline \multirow{3}{*}{ Genotypes of cultivars ${ }^{\mathrm{a}}$} & \multicolumn{3}{|c|}{ Testers genotype } \\
\hline & $\begin{array}{l}\text { W26,W3,W2 } \\
(h w d 1 / h w d 1 \\
h w d 2 / h w d 2)\end{array}$ & $\begin{array}{c}\text { Sasanishiki } \\
(H w d 1 / H w d 1 \\
h w d 2 / h w d 2)\end{array}$ & $\begin{array}{l}\text { Col. No. } 15 \\
(h w d 1 / h w d 1 \\
H w d 2 / H w d 2)\end{array}$ \\
\hline & Plant type of $\mathrm{F}_{1}$ & Segregation in $\mathrm{F}_{2}^{\mathrm{b}}$ & Segregation in $\mathrm{F}_{2}^{\mathrm{b}}$ \\
\hline$H w d 1 / H w d 1$ Hwd2 / Hwd2 & Normal & No & No \\
\hline$H w d 1 / H w d 1$ hwd2/hwd2 & Weak & No & Yes \\
\hline$h w d 1 / h w d 1 \mathrm{Hwd} / \mathrm{Hwd} 2$ & Weak & Yes & No \\
\hline
\end{tabular}

were classified as unidentified.

Evaluation of the genetic diversity of the chromosomes harboring hwd1 and hwd2. The genes $h w d 1$ and $h w d 2$ are located on chromosomes 10 and 7 , respectively (Fukuoka et al., 1998). To analyze the genetic diversity in the regions harboring $h w d 1$ and $h w d 2$, RFLP markers located on the chromosomes 10 and 7 were surveyed in 160 cultivars, 93 belonging to group I and 67 belonging to group II. Group III and unclassified cultivars were not used in this analysis. Southern hybridization was conducted with 12 RFLP probes (12 loci) on chromosome 10 and 33 RFLP probes (34 loci) on chromosome 7. For alleles at each of the RFLP marker loci, the proportion of group I and group II cultivars were calculated. Based on these proportions, each allele was determined as to whether it was specific to group I or group II. The agreement between the classification by the three diagnostic RFLP markers (C601, G187, and C711) and the classification by single RFLP marker was calculated for each locus. The percentage thus obtained was regarded as indicating the degree of association of each locus with the classification of cultivars into indica and japonica.

\section{RESULTS}

Genotype determination and geographical variation of $\boldsymbol{h w d 1}$ and $\boldsymbol{h w d 2 . ~ T h e ~ g e n o t y p e s ~ f o r ~} h w d 1$ and $h w d 2$ were determined by test crosses (Table 3). Fifty-three

Table 3. Genotype classification for $h w d 1$ and $h w d 2$ of cultivars

\begin{tabular}{|c|c|c|c|c|c|c|c|c|c|c|c|c|c|c|}
\hline & Genotype & \multicolumn{3}{|c|}{$\begin{array}{l}H w d 1 / H w d 1 \\
H w d 2 / H w d 2\end{array}$} & \multicolumn{3}{|c|}{$\begin{array}{l}H w d 1 / H w d 1 \\
h w d 2 / h w d 2\end{array}$} & \multicolumn{3}{|c|}{$\begin{array}{l}\text { hwd1 / hwd1 } \\
H w d 2 / H w d 2\end{array}$} & \multicolumn{3}{|c|}{ unidentified } & \multirow[b]{2}{*}{ total } \\
\hline & Cultivar group* & I & II & III & I & II & III & I & II & III & I & II & III & \\
\hline Code No. & Country/ Area & \multirow[b]{2}{*}{9} & \multirow[b]{2}{*}{0} & \multirow[b]{2}{*}{1} & \multirow[b]{2}{*}{1} & \multirow[b]{2}{*}{1} & \multirow[b]{2}{*}{0} & \multirow[b]{2}{*}{0} & \multirow[b]{2}{*}{0} & \multirow[b]{2}{*}{0} & \multirow[b]{2}{*}{1} & \multirow[b]{2}{*}{0} & \multirow[b]{2}{*}{0} & \multirow[b]{2}{*}{13} \\
\hline 1 & Sri Lanka & & & & & & & & & & & & & \\
\hline 2 & India & 7 & 0 & 2 & 1 & 0 & 0 & 0 & 0 & 0 & 1 & 0 & 0 & 11 \\
\hline 3 & Bangladesh & 6 & 0 & 1 & 4 & 0 & 1 & 0 & 0 & 0 & 2 & 0 & 0 & 14 \\
\hline 4 & India (Assam) & 5 & 0 & 1 & 5 & 0 & 0 & 0 & 0 & 0 & 6 & 0 & 0 & 17 \\
\hline 5 & Nepal & 8 & 0 & 0 & 1 & 1 & 0 & 0 & 0 & 0 & 1 & 1 & 0 & 12 \\
\hline 6 & Bhutan & 3 & 4 & 0 & 1 & 2 & 0 & 0 & 0 & 0 & 0 & 3 & 0 & 13 \\
\hline 7 & Myanmar & 11 & 1 & 0 & 0 & 0 & 0 & 0 & 0 & 0 & 1 & 0 & 0 & 13 \\
\hline 8 & Thailand & 5 & 3 & 1 & 0 & 2 & 0 & 0 & 0 & 0 & 0 & 1 & 0 & 12 \\
\hline 9 & Cambodia & 10 & 0 & 0 & 0 & 0 & 0 & 0 & 0 & 0 & 1 & 0 & 0 & 11 \\
\hline 10 & Laos & 4 & 4 & 0 & 0 & 1 & 0 & 1 & 0 & 0 & 0 & 1 & 0 & 11 \\
\hline 11 & Vietnam (northwest) & 2 & 5 & 0 & 0 & 1 & 0 & 0 & 0 & 0 & 0 & 3 & 0 & 11 \\
\hline 12 & Malaysia & 5 & 0 & 0 & 0 & 5 & 0 & 0 & 0 & 0 & 1 & 1 & 0 & 12 \\
\hline 13 & Indonesia & 5 & 0 & 2 & 0 & 7 & 0 & 0 & 0 & 0 & 0 & 0 & 0 & 14 \\
\hline 14 & Philippines & 2 & 1 & 0 & 0 & 10 & 0 & 0 & 0 & 0 & 2 & 2 & 1 & 18 \\
\hline 15 & China (Yunnan) & 5 & 0 & 0 & 0 & 6 & 0 & 0 & 0 & 0 & 0 & 2 & 0 & 13 \\
\hline 16 & China (south) & 7 & 1 & 0 & 0 & 1 & 0 & 0 & 0 & 0 & 6 & 0 & 0 & 15 \\
\hline 17 & China (north) & 4 & 2 & 0 & 0 & 5 & 0 & 0 & 0 & 0 & 3 & 1 & 0 & 15 \\
\hline 18 & Japan & 0 & 0 & 0 & 0 & 13 & 0 & 0 & 0 & 0 & 0 & 1 & 0 & 14 \\
\hline & total & 98 & 21 & 8 & 13 & 55 & 1 & 1 & 0 & 0 & 25 & 16 & 1 & 239 \\
\hline & & & 127 & & & 69 & & & 1 & & & 42 & & \\
\hline
\end{tabular}

* Cultivar group was determined based on three diagnostic RFLP markers; C601, G187, and C711. Group I and II correspond to indica and japonica, respectively. Group III is an intermediate type based on the three RFLP markers. 
percent of cultivars tested had the genotype Hwd1 / Hwd1 Hwd2 /Hwd2 and 29\% had Hwd1/Hwd1 hwd2/hwd2. The genotype hwd1/hwd1 Hwd2/Hwd2 was rare and found in only one cultivar. The genotype of $18 \%$ of the cultivars could not be determined due to unexpected results of the three test crosses. The frequencies of the cultivars with the genotypes Hwd1/Hwd1 Hwd2 / Hwd2, Hwd1 / Hwd1 hwd2 / hwd2 and hwd1 / hwd1 Hwd2 / Hwd2 were $64.5 \%, 35.0 \%$, and $0.5 \%$, respectively, when unidentified samples were excluded.

The distribution of genotypes revealed geographical variation in the frequency of $h w d 1$ and $h w d 2$ (Fig. 1). The frequency for the cultivar with the genotype Hwd1 / Hwd1 Hwd2 /Hwd2 was highest in mainland Southeast Asian countries such as Cambodia, Myanmar, and Thailand. Those with the genotype Hwd1/Hwd1 hwd2 / hwd2 were mainly found in cultivars of Yunnan province of China, the
Philippines, and Japan. The cultivars with both genotypes were found in all countries and regions except Japan. The genotype hwd1/hwd1 Hwd2 / Hwd2, which was first found in Col. No. 15, was only found in one other cultivar from Laos in this survey. Therefore, we can conclude that differentiation of the genetic system controlling this hybrid breakdown in Asian cultivated rice mainly occurred at the hwd2 locus.

Relationship between cultivar groups classified by RFLP markers and genotypes for hybrid breakdown. Cultivars for which respective hybrid breakdown genotypes were determined were further classified into groups I, II, and III based on RFLP (Table 3). Seventy-seven percent and $17 \%$ of the cultivars with the genotype Hwd1/Hwd1 Hwd2 / Hwd2 belonged to groups I and II, respectively. However, $19 \%$ and $80 \%$ of the cultivars with the genotype

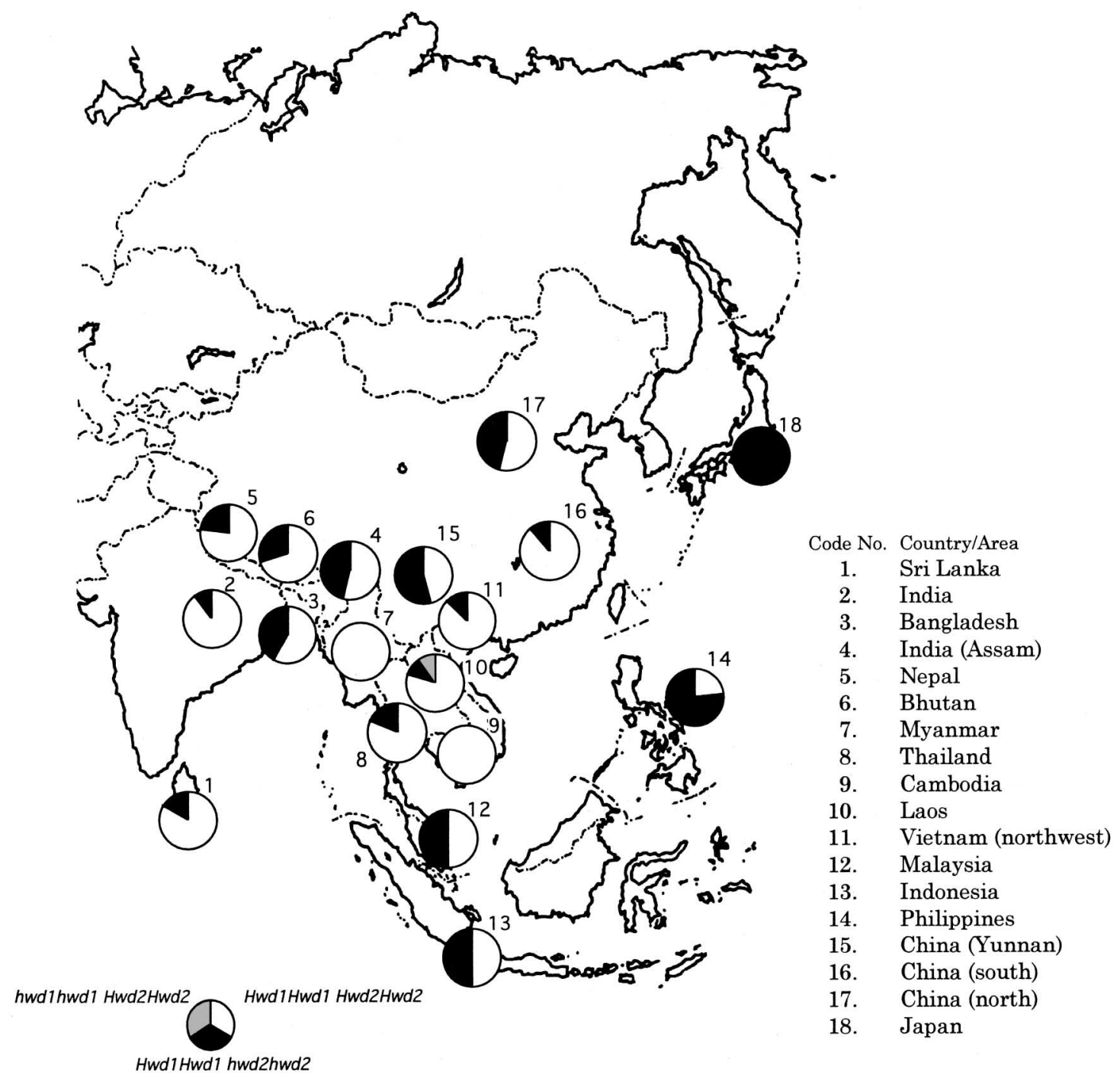

Fig. 1. Geographical variation in the genotypes for $h w d 1$ and $h w d 2$ in Asian rice cultivars. The cultivars with unidentified genotypes have been excluded. Countries and areas are indicated with code numbers. The names of countries and areas are shown on the right. 
Hwd1 / Hwd1 hwd2 / hwd2 belonged to groups I and II, respectively. This indicates preferential association between the cultivar groups defined by the allelic combination at the diagnostic RFLP markers and the $h w d 2$ locus. This association was not found in cultivars from Bangladesh, Bhutan, Laos and Vietnam (northwest). Only one cultivar Tamen from Laos with the hwd1/ hwd1 Hwd2 / Hwd2 genotype was classified as group I. The cultivars unidentified

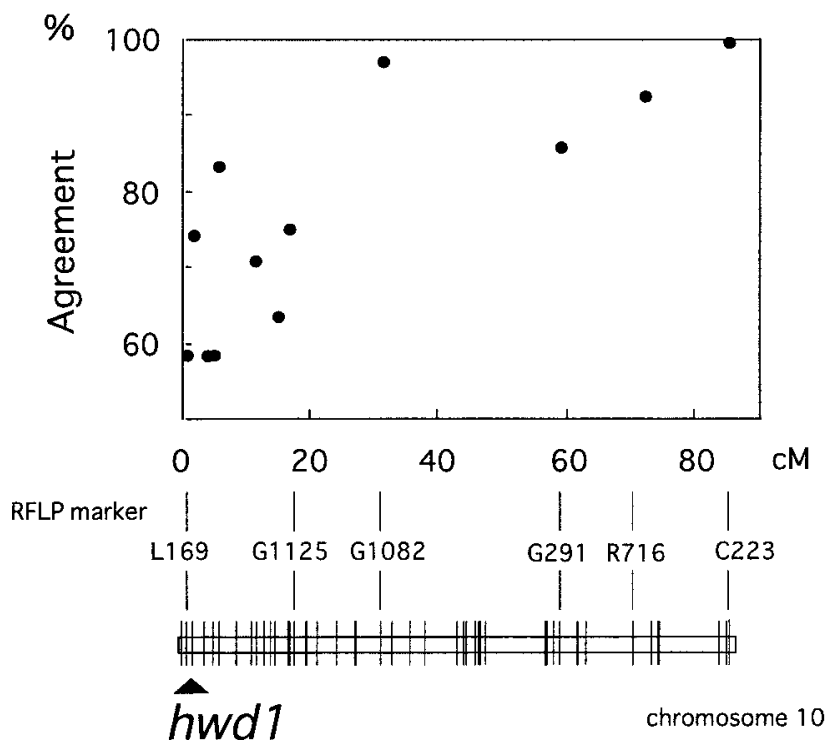

Fig. 2. Percentages of agreement of the RFLP markers distributed along a genetic linkage map of chromosome 10 with the cultivar-group classification. The frame of the genetic linkage map of chromosome 10 (Kurata et al., 1994) is shown below and position of $h w d 1$ is indicated with an arrow. for the $h w d 1$ and $h w d 2$ loci were found most frequently in cultivars from south China and Assam, India.

Genetic differentiation of the chromosomes harboring hybrid breakdown genes. Genetic differentiation around the chromosomal regions harboring $h w d 1$ and $h w d 2$ were analyzed. Percentages of agreement between classification into cultivar groups I and II and the classification by each RFLP marker on chromosomes 7 and 10 were plotted along the respective linkage maps (Figs. 2 and 3 ). The percentages ranged from 58 to $100 \%$. A low percentage at a given locus indicates that this region is not involved in intraspecific differentiation, while a high percentage indicates the region may possibly have a relationship with intraspecific differentiation. On chromosome 10 , the percentage of agreement was low around the $h w d 1$ locus, though it was higher in other regions on this chromosome. However, most of the RFLP marker loci on chromosome 7 had high percentages of agreement with classification into cultivar groups. The percentages were higher than $80 \%$ for the 12 RFLP marker loci closest to $h w d 2$.

\section{DISCUSSION}

In the present study, the geographical variation of $h w d 1$ and $h w d 2$ and genetic differentiation in the chromosomal regions harboring $h w d 1$ and $h w d 2$ were analyzed to clarify the significance of hybrid breakdown $\left(\mathrm{F}_{2}\right.$ weakness $)$ in relation to the differentiation of Asian rice cultivars.

Geographical variation was observed in the distribution of hwd2. Hwd2 was predominant in South Asia and

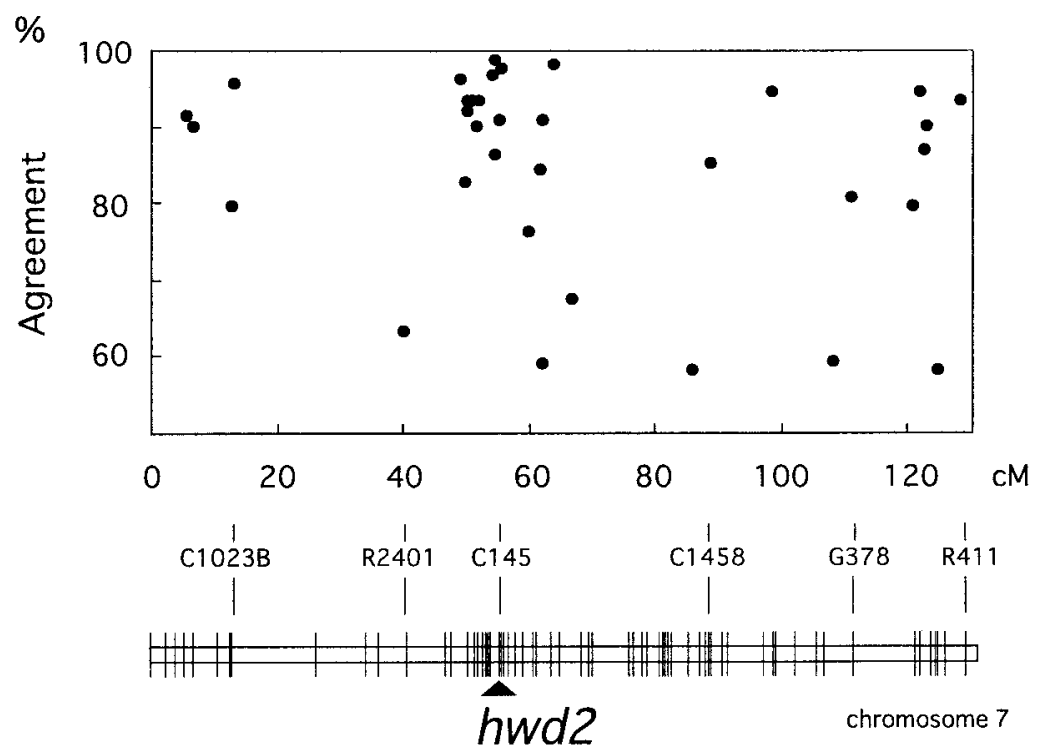

Fig. 3. Percentages of agreement of the RFLP markers distributed along a genetic linkage map of chromosome 7 with the cultivar-group classification. The frame of genetic linkage map of chromosome 7 (Kurata et al., 1994) is shown below and position of $h w d 2$ is indicated with an arrow. 
Southeast Asia, while $h w d 2$ was predominant in Japan and the Philippines. Dicotomy in the genotypes between two regions was reported for the $H w c 2$ locus associated with $\mathrm{F}_{1}$ weakness (Sato and Hayashi, 1983) and the hca2 locus associated with $\mathrm{F}_{2}$ chlorosis (Sato and Morishima, 1988). Using a double recessive tester line, Okuno (1986) previously reported that the frequency of cultivars with the genotype $H w d 1 / H w d 1 H w d 2 / H w d 2$ was high in South China, Vietnam, Laos, Myanmar, and Thailand. Similar results in the present study were obtained using a different cultivar set. Sixty percent of the cultivars had this double dominant genotype in the present study, while $30 \%$ of the cultivars had this genotype in the previous study. The difference in frequency can be explained by the sample choice. The present materials included more samples from South and Southeast Asia, where the cultivars with $H w d 1 / H w d 1 H w d 2$ / Hwd2 are commonly found.

The present study revealed that cultivar with the genotype $h w d 1 / h w d 1 \mathrm{Hwd} / \mathrm{Hwd} 2$ was rare. A level of low polymorphism at one of the loci for $F_{1}$ weakness and $F_{2}$ chlorosis has also been reported in rice and other crops (Sato and Hayashi, 1983; Takahashi and Hayashi, 1987; Konishi, 1987; Sato and Morishima, 1988). So far, the genotype $h w d 1$ / hwd1 Hwd2 / Hwd2 has been found in only one cultivar from Laos and Col. No. 15 from Thailand. Since they seem to have a close genetic relationship based on RFLP analyses, the allele $h w d 1$ found in these cultivars might have a common origin. These observations support the hypothesis that hybrid breakdown controlled by hwd1 and $h w d 2$ did not cause or promote intraspecific differentiation.

Using three testers, we could not determine the genotypes of $18 \%$ of the cultivars for $h w d 1$ and $h w d 2$. There are three possible explanations for this result. One is misjudgment of the $F_{1}$ phenotypes in the cross with the recessive tester line. Some $F_{1}$ plants had intermediate plant types and it was difficult to judge their phenotypes. A second possible explanation is the effect of unidentified genes causing similar $\mathrm{F}_{1}$ weakness or hybrid breakdown. A third explanation is heterogeneity within a cultivar at the $h w d 1$ or $h w d 2$ locus. Since the heterogeneous cultivars in the chromosomal regions harboring $h w d 1$ and $h w d 2$, which were detected by RFLP analysis, were excluded from this study, the third explanation is unlikely. One or more of these three possible explanations may be involved in the difficulty in determining the genotypes for hybrid breakdown in some cultivars.

A clear association was observed between the genotype at the $h w d 2$ locus and the cultivar group to which the cultivar belonged. Seventy-seven percent of cultivars with the Hwd1 / Hwd1 Hwd2 / Hwd2 genotype belonged to group I (indica) and $80 \%$ of cultivars with the $H w d 1 / H w d 1$ $h w d 2 / h w d 2$ genotype belonged to group II (japonica). A similar preferential association with indica and japonica groups was found in the analysis of the $F_{1}$ weakness gene hwc2 (Sato and Hayashi, 1983). In the present study, the association was obscure in some areas such as northwest Vietnam and Laos. These areas are included in the center of the genetic diversity of rice revealed by esterase isozyme analyses (Nakagahra et al., 1975). The present study also suggests that the cultivars in these regions are more genetically diverse than those in the other regions (Table 3 ).

The present study confirms that RFLPs on chromosome 7 , especially in the region around $h w d 2$, could be used with a high percentage agreement with the classification into two cultivar groups, indica and japonica. This suggests that some genes responsible for indica/japonica differentiation are located in this region, although it is unclear what genes they are. QTLs concerned with agronomically important traits such as plant height and panicle length are located around the region of $h w d 2$ in an indica/japonica cross (Xiao et al., 1996; Li et al., 1998). Such observations suggest the involvement of this region in indica/japonica differentiation because the genes concerned with agronomic traits would be easily selected.

The present study revealed that hybrid breakdown $\left(\mathrm{F}_{2}\right.$ weakness) is a rare event and therefore, there would be little chance that this phenomenon would impede varietal improvement by cross breeding. Also the present study implies that the polymorphic locus $h w d 2$, one of the hybrid breakdown loci, is located in a chromosomal region possibly associated with differentiation in Asian cultivated rice. However, it may be concluded that the genes for hybrid breakdown investigated in this study are not a factor causing genetic differentiation in rice.

The authors thank H. Morishima and D. A. Vaughan for their critical reading of the manuscript and accurate comments. This research was supported by the project, development of methods of rice genome analysis and use of accurate genome map of rice from the Ministry of Agriculture, Forestry, and Fisheries of Japan.

\section{REFERENCES}

Chu, Y. E., and Oka, H. I. (1972) The distribution and effect of genes causing $\mathrm{F}_{1}$ weakness in Oryza breviligulata and $O$. glaberrima. Genetics 70, 163-173.

Fukuoka, S., Namai, H., and Okuno, K. (1998) RFLP mapping of the genes controlling hybrid breakdown in rice (Oryza sativa L.). Theor. Appl. Genet. 97, 446-449.

Ise, K., Sekizawa, K., and Sato, H. (1992) Inheritance of hybrid weakness in indica/japonica rice crosses. Int. Rice Res. Notes 17-4, 5 .

Konishi, T. (1987) Genetic studies on hybrid weakness in barley with special reference to phylogenetic differentiation. Barley Genet. 5, 145-153.

Kurata, N., Nagamura, Y., Yamamoto, K., Harushima, Y., Sue, N., Wu, J., Antonio, B. A., Shomura, A., Shimizu, T., Lin, S. Y., Inoue, T., Fukuda, A., Shimano, T., Kuboki, Y., Toyama, T., Miyamoto, Y., Kirihata, T., Hayasaka, K., Miyao, A., Monna, L., Zhong, H.S., Tamura, Y., Wang, Z. X., Momma, T., Umehara,Y., Sasaki, T., and Minobe,Y. (1994) A 330 kilobase interval genetic map of rice including 883 expressed sequences. Nat. Genet. 8, 365-372. 
Li, R. H., Xue, C. G., Yuan, L. P.,He, Y. Q., Sun, C. Q., Yu, S. B., Li, X. H., and Wang, X. K. (1998) Differentiation and classification of parental lines and favorable genic interactions affecting $\mathrm{F}_{1}$ fertility in distant crosses of rice (Oryza sativa L.). Theor. Appl. Genet. 96, 526-538.

Nakagahra, M., Akihama, T., and Iwata, N. (1975) Genatic variation and geographic cline of esterase isozyme in native rice varieties. Jpn. J. Genet. 50, 373-382.

Oka, H. I. (1957a) Genic analysis for the sterility of hybrids between distantly related varieties of cultivated rice. J. Genet. 55, 397-409.

Oka, H. I. (1957b) Phylogenetic differentiation of cultivated rice. XV. Complementary lethal genes in rice. Jpn. J. Genet. 32, 83-87.

Oka, H. I. (1974) Analysis of genes controlling $\mathrm{F}_{1}$ sterility in rice by use of isogenic lines. Genetics 77, 521-534.

Okuno, K. (1985) Complementary recessive genes controlling hybrid breakdown found in a varietal cross of rice. Rice Genet. Newsl. 2, 52-54.

Okuno, K. (1986) Geographical distribution of complementary recessive genes controlling hybrid breakdown of rice. Rice Genet. Newsl. 3, 44-45.

Qian, H-R., Zhuang, J-Y., Lin, H-X., Lu, J., and Zheng, K-L. (1995) Identification of a set of RFLP probes for subspecies differentiation in Oryza sativa L. Theor. Appl. Genet. 90, 878-884.

Sano, Y., Chu, Y. E., and Oka, H. I. (1979) Genetic studies of speciation in cultivated rice, 1 . Genic analysis for $\mathrm{F}_{1}$ sterility between Oryza sativa L. and O. glaberrima Steud. Jpn. J. Genet. 54, 121-132.
Sato, Y-I., and Hayashi, K. (1983) Distribution of the complementary genes causing $F_{1}$ weakness in the common rice and its wild relatives. I. $L-2-a$ gene in Asian native cultivars. Jpn. J. Genet. 58, 411-418.

Sato, Y. I., Matsuura, S., and Hayashi, K. (1984) The genetic basis of hybrid chlorosis found in a cross between Japanese native cultivars. Rice Genet. Newsl. 1, 106-107.

Sato, Y. I., and Morishima, H. (1987) Studies on the distribution of complementary genes causing $\mathrm{F}_{1}$ weakness in common rice and its wild relatives. 2. Distribution of two comlementary genes, Hwd-1 and Hwc-2 gene in native cultivars and its relatives of tropical Asia. Euphytica 36, 425-431.

Sato, Y. I., and Morishima, H. (1988) Distribution of the genes causing $\mathrm{F}_{2}$ chlorosis in rice cultivars of the Indica and Japonica types. Theor. Appl. Genet. 75, 723-727.

Stebbins, G. L. (1950) Isolation and the origine of species. In: Variation and Evolution in Plants (ed.: G. L. Stebbins, Jr.), pp. 189-250. Columbia Univ. Press, New York.

Takahashi, R., and Hayashi, J. (1987) Studies on chlorotic plants of barley by dominant complementary genes and geographical distribution of the genes concerned. Barley Genet. 5, 139-144.

Xiao, J., Li, J., Yuan, L., and Tanksley, S. D. (1996) Identification of QTLs affecting traits of agronomic importance in a recombinant inbreed population derived from a subspecific rice cross. Theor. Appl. Genet. 92, 230-244.

Zheng, K., Quian, H., Shen B., Lin, H., and Lu, J. (1994) RFLPbased phylogenetic analysis of wide compatibility varieties in Oryza sativa L. Theor. Appl. Genet. 88, 65-69. 